\title{
SOME COEFFICIENT INEQUALITIES AND DISTORTION BOUNDS ASSOCIATED WITH CERTAIN NEW SUBCLASSES OF ANALYTIC FUNCTIONS
}

\author{
Shigeyoshi Owa, Kyohei Ochiai AND H. M. SRIVAStaVA
}

\begin{abstract}
The authors introduce and investigate two new subclasses $\mathscr{M}^{*}(\alpha)$ and $\mathscr{N}^{*}(\alpha)$ of normalized analytic functions satisfying certain coefficient inequalities in the open unit disk $\mathbb{U}$. The main results of the present paper provide various interesting properties of functions belonging to the classes $\mathscr{M}^{*}(\alpha)$ and $\mathscr{N}^{*}(\alpha)$. Some of these properties include (for example) several coefficient inequalities, distortion bounds, and inclusion relationships for the function classes which are considered here.
\end{abstract}

Mathematics subject classification (2000): 30C45, 30A10, 30C50.

Key words and phrases: Coefficient inequalities, distortion bounds, analytic functions, univalent functions, starlike functions, convex functions, integro-differential operator, inclusion relationships, monotonically increasing and monotonically decreasing functions.

\section{REFERENCES}

[1] P. L. DuREN, Univalent Functions, Grundlehren der Mathematischen Wissenschaften 259, SpringerVerlag, New York, Berlin, Heidelberg and Tokyo, 1983.

[2] A. W. Goodman, Univalent Functions, Vol. I, Polygonal Publishing House, Washington, New Jersey, 1983.

[3] S. Owa, H. M. SRIVASTAVA AND N. Saito, Partial sums of certain classes of analytic functions, Internat. J. Comput. Math., 81, (2005), 1239-1256.

[4] T. SEKINE, S. OwA, Certain subclasses of starlike functions of order $\alpha$, Pan Amer. Math. J., 5, 1 (1995), 95-100.

[5] H. Silverman, Univalent functions with negative coefficients, Proc. Amer. Math. Soc., 51, (1975), 109-116.

[6] H. M. SRivastava, S. Owa (Editors), Current Topics in Analytic Function Theory, World Scientific Publishing Company, Singapore, New Jersey, London and Hong Kong, 1992. 